TRANSACTIONS OF THE

AMERICAN MATHEMATICAL SOCIETY

Volume 358, Number 7 , Pages 3061-3075

S 0002-9947(05)03778-5

Article electronically published on September 22, 2005

\title{
FINITE BRUCK LOOPS
}

\author{
MICHAEL ASCHBACHER, MICHAEL K. KINYON, AND J. D. PHILLIPS
}

\begin{abstract}
Bruck loops are Bol loops satisfying the automorphic inverse property. We prove a structure theorem for finite Bruck loops $X$, showing that $X$ is essentially the direct product of a Bruck loop of odd order with a 2-element Bruck loop. The former class of loops is well understood. We identify the minimal obstructions to the conjecture that all finite 2-element Bruck loops are 2-loops, leaving open the question of whether such obstructions actually exist.
\end{abstract}

Let $X$ be a magma; that is, $X$ is a set together with a binary operation $\circ$ on $X$. For each $x \in X$ we obtain maps $R(x)$ and $L(x)$ on $X$ defined by $R(x): y \mapsto y \circ x$ and $L(x): y \mapsto x \circ y$ called right and left translation by $x$, respectively. A loop is a magma $X$ with an identity 1 such that $R(x)$ and $L(x)$ are permutations of $X$ for all $x \in X$. In essence loops are groups without the associative axiom. See $[\mathrm{Br}]$ for further discussion of basic properties of loops.

Certain classes of loops have received special attention: A loop $X$ is a (right) Bol loop if it satisfies the (right) Bol identity (Bol) for all $x, y, z \in X$ :

$$
((z \circ x) \circ y) \circ x=z \circ((x \circ y) \circ x) .
$$

In a Bol loop, the subloop $\langle x\rangle$ generated by $x \in X$ is a group. Thus we can define $x^{-1}$ and the order $|x|$ of $x$ to be the inverse of $x$ and the order of $x$ in that group.

The loop $X$ satisfies the AIP-property if $(x \circ y)^{-1}=x^{-1} \circ y^{-1}$ for all $x, y \in X$. Finally $X$ is a Bruck loop if $X$ is a Bol loop satisfying the AIP-property.

We prove many of our results on loops by translating them into results about groups, using an observation of Reinhold Baer in Ba]: Given a loop $X$, define $K=\{R(x): x \in X\}$, regarded as a subset of the symmetric group $\operatorname{Sym}(X)$ on $X, G=\langle K\rangle$, to be the subgroup of $\operatorname{Sym}(X)$ generated by $K$, and $H=G_{1}$ to be the stabilizer in $G$ of the identity 1 of $X$. Set $\epsilon(X)=(G, H, K)$. We call $G$ the enveloping group of $X, H$ the (right) inner mapping group of $X$, and $\epsilon(X)$ the envelope of $X$.

The loop $X$ is said to be an $A_{r}$-loop if $H$ acts as a group of automorphisms of $X$ in its representation on $X$.

We can now state our main theorems.

Received by the editors December 15, 2003 and, in revised form, June 29, 2004.

2000 Mathematics Subject Classification. Primary 20N05.

The first author was partially supported by NSF-0203417. 
Theorem 1. Let $X$ be a finite Bruck loop and let $G$ be the enveloping group of $X$. Then:

(1) $X=O^{2^{\prime}}(X) * O(X)$ and $G=O^{2^{\prime}}(G) * O(G)$ are central products.

(2) $O^{2^{\prime}}(X) \cap O(X)=Z$ is a subloop of $Z(X)$ of odd order and $O^{2^{\prime}}(G) \cap O(G)$ is a subgroup of $Z(G)$ of odd order.

(3) $X / Z=O^{2^{\prime}}(X) / Z \times O(X) / Z$.

(4) $O^{2}(X)=O(X)$, so $O^{2^{\prime}}(X) / Z$ is a 2-element loop.

(5) If $X$ is solvable, then $O^{2^{\prime}}(X)=O_{2}(X)$, so $X=O_{2}(X) \times O(X)$ and $G=$ $\mathrm{O}_{2}(G) \times O(G)$.

See [FGT] for notation and terminology involving groups. We write $O_{2}(X)$, $O(X)$ for the largest normal subloop of $X$ of order a power of 2 , odd order, respectively. Further $O^{2^{\prime}}(X), O^{2}(X)$ denotes the subloop generated by all 2-elements of $X$ (i.e. elements of order a power of 2), $2^{\prime}$-elements (elements of odd order), respectively. Finally $X$ is a 2-element loop if every element of $X$ is a 2-element.

One of the main tools in the proof of Theorem 1 is the following result about arbitrary Bruck loops, which is of independent interest.

Theorem 2. Let $X$ be a Bruck loop and let $x, y \in X$ with $x$ a 2-element and $y$ an element of odd order. Then $R(x) R(y)=R(x \circ y)=R(y \circ x)=R(y) R(x)$. Hence $x \circ y=y \circ x$.

Theorem 1 reduces the study of finite Bruck loops to the study of 2-element loops and loops of odd order. The category of Bruck loops of odd order is essentially equivalent to the category of pairs $(G, \tau)$, where $G$ is a group of odd order and $\tau$ an involutory automorphism of $G$ such that $G=[G, \tau]$ and $C_{Z(G)}(\tau)=1$. This fact goes back to Glauberman in G2 and [G3]; see also 5.7, 5.8, and 5.10. As a result, Bruck loops of odd order are well behaved and well understood. On the other hand Bruck 2-loops seem difficult to analyze.

It seems possible that all finite Bruck loops $X$ are solvable, and hence $X=$ $\mathrm{O}_{2}(X) \times O(X)$. Our next theorem is a step toward proving that finite Bruck loops are indeed solvable. Define a finite Bruck loop to be an $M$-loop if each proper section of $X$ is solvable, but $X$ is not solvable.

Theorem 3. Let $X$ be an $M$-loop, let $\epsilon(X)=(G, H, K)$, let $J=O_{2}(G)$, and let $G^{*}=G / J$. Then:

(1) $X$ is a simple 2-element loop.

(2) $G^{*} \cong P G L_{2}(q)$, with $q=2^{n}+1 \geq 5, H^{*}$ is a Borel subgroup of $G^{*}$, and $K^{*}$ consists of the involutions in $G^{*}-F^{*}\left(G^{*}\right)$.

(3) $F^{*}(G)=J$.

(4) Let $n_{0}=|K \cap J|$ and $n_{1}=|K \cap a J|$ for $a \in K-J$. Then $n_{0}$ is a power of 2, $n_{0}=n_{1} 2^{n-1}$, and $|X|=|K|=(q+1) n_{0}=n_{1} 2^{n}\left(2^{n-1}+1\right)$.

One would like to show that M-loops do not exist, and hence show that finite Bruck loops are solvable. Theorem 3 identifies a set of obstructions to that goal. This is essentially the same set of obstructions to the Main Theorem of [A2] on Bol loops of exponent 2. See section 12 of [A2] for a discussion of possible approaches to eliminating these obstructions or alternatively to constructing examples of M-loops. These approaches involve the analysis of Bruck 2-loops.

The class of finite Bol loops which are also $A_{r}$-loops is much larger than the class of finite Bruck loops; for example the former class includes all finite groups. Still 
(cf. Lemma 8.1) the latter class can be described in terms of the former class and the class of finite groups, allowing us to prove:

Corollary 4. Let $X$ be a finite loop which is both a Bol loop and an $A_{r}$-loop. Then $X$ is solvable iff the enveloping group of $X$ is solvable.

The proof of Theorem 1 uses the solvability of groups of odd order, established by Feit and Thompson [FT], Glauberman's $Z^{*}$-Theorem [G1], and several other results from the theory of finite groups, whose proofs are a bit easier and can be found in [FGT]. The proof of Theorem 3 involves appeals to the Main Theorem of [A2] and its proof, which in turn uses the classification of the finite simple groups, together with deep knowledge of the subgroup structure of the automorphism groups of those groups.

\section{LOOPS, FOLDERS, ENVELOPES, AND TWISTED SUBGROUPS}

In this section we recall some notation and terminology involving loops, summarize some facts about loops, and references for those facts.

In [A2, a loop folder is defined to be a triple $\xi=(G, H, K)$, where $G$ is a group, $H$ is a subgroup of $G, K$ is a subset of $G$ containing 1 , and for all $g \in G, K$ is a set of coset representatives for $H^{g}$ in $G$. The folder is an envelope if $G=\langle K\rangle$ and faithful if $\operatorname{ker}_{H}(G)=1$, where $\operatorname{ker}_{H}(G)$ is the largest normal subgroup of $G$ contained in $H$.

For example if $X$ is a loop, then $\epsilon(X)$ is a faithful loop envelope.

Section 1 of A2 contains the definition of a category of loop folders and functors $\epsilon$ and $l$ to and from the category of loops and the category of loop folders. The reader is directed to A2 for notation, terminology, and results about folders and these functors.

A twisted subgroup of a group $G$ is a subset $K$ of $G$ such that $1 \in K$ and for all $x, y \in K, x y^{-1} x \in K$. See section 5 of A2 for a brief discussion of twisted subgroups taken from A1.

A folder $\xi=(G, H, K)$ is a Bol loop folder if $K$ is a twisted subgroup of $G$. Further (cf. 6.1 in A2 ]) a loop $X$ is a Bol loop iff $\epsilon(X)=(G, H, K)$ is a Bol folder. In that event there is a normal subgroup $\Xi_{K}(G)$ of $G$ called the $K$-radical of $G$, and a corresponding normal subloop $\Xi(X)$ of $X$ (which is a group) called the radical of $X$. Moreover if $\Xi_{K}(G)=1$, then there is a unique automorphism $\tau=\tau_{X}$ of $G$ such that $\tau^{2}=1$ and $K \subseteq K(\tau)$, where

$$
K(\tau)=\left\{g \in G: g^{\tau}=g^{-1}\right\} .
$$

See section 6 of $\mathrm{A} 2$ for further discussion.

Next $X$ is an $A_{r}$-loop iff $H$ acts on $K$ via conjugation (cf. 4.1 in A2 ). Further $X$ is a Bruck loop iff $X$ is a radical free (i.e. $\Xi(X)=1) A_{r}$-loop (cf. 6.7 in A2]).

The material in the remainder of this section is elementary and easy. See for example section 1 of chapter IV in $\mathrm{Br}$ for more discussion and proofs.

A normal subloop of a loop $X$ is the kernel of a loop homomorphism. Further a subloop $Y$ of $X$ is normal iff for all $a, b \in X$,

$$
a \circ(Y \circ b)=Y \circ(a \circ b)=(a \circ Y) \circ b,
$$

in which case the cosets $Y \circ x, x \in X$, form the equivalence classes of an equivalence relation on $X$, and we can form the factor loop $X / Y$ on this set of equivalence 
classes, with multiplication defined by

$$
(X \circ a) \circ(X \circ b)=X \circ(a \circ b) .
$$

Also we obtain the surjective loop homomorphism $\pi: X \rightarrow X / Y$ with $x \pi=Y \circ x$ and $\operatorname{ker}(\pi)=Y$. We have the usual facts:

(1.1) If $\varphi: X \rightarrow X^{\prime}$ is a surjective loop homomorphism with $\operatorname{ker}(\varphi)=Y$, then:

(1) $\psi: X / Y \rightarrow X^{\prime}$ defined by $(Y \circ x) \psi=x \varphi$ is an isomorphism with $\pi \psi=\varphi$.

(2) If $U \unlhd X$, then $U \varphi \unlhd X^{\prime}$.

(3) If $V \unlhd X^{\prime}$, then $V \varphi^{-1} \unlhd X$.

\section{Normal StruCture OF LOOPS}

In this section $\xi=(G, H, K)$ is a loop envelope and $X=l(\xi)$.

(2.1) Let $\xi_{i}=\left(G_{i}, H_{i}, K_{i}\right)$ be normal subfolders of $\xi$, set $\xi^{i}=\xi / \xi_{i}$, and let $\pi_{i}$ : $\xi \rightarrow \xi^{i}$ be the natural map of 2.6 in A2 with $\operatorname{ker}\left(\pi_{i}\right)=\xi_{i}$. Then:

(1) $\xi_{3-i} \pi_{i} \unlhd \xi^{i}$.

(2) Let $G_{3}=G_{1} G_{2}, H_{3}=H \cap G_{3}$, and $K_{3}=K \cap G_{3}$. Then $\xi_{3}=\left(G_{3}, H_{3}, K_{3}\right) \unlhd$ $\xi$ and $\xi_{3} \pi_{i}=\xi_{3-i} \pi_{i}$.

(3) $\xi / \xi_{3} \cong \xi^{i} / \xi_{3-i} \pi_{i}$.

(4) Let $G_{0}=G_{1} \cap G_{2}, H_{0}=H_{1} \cap H_{2}$, and $K_{0}=K_{1} \cap K_{2}$. Then $\xi_{0} \unlhd \xi$.

(5) Set $\bar{G}=G / G_{0}$. Then $\bar{G}_{3}=\bar{G}_{1} \times \bar{G}_{2}, \bar{H}_{3}=\bar{H}_{1} \times \bar{H}_{2}$, and $\bar{K}_{3}=\bar{K}_{1} \times \bar{K}_{2}$.

(6) Let $X_{i}=l\left(\xi_{i}\right)$. Then $X_{i}$ is normal in $X$ for each $i$ and $X_{3} / X_{0} \cong X_{1} / X_{0} \times$ $X_{2} / X_{0}$.

Proof. Let $G^{*}=G \pi_{1}$. Then $\xi^{1}=\left(G^{*}, H^{*}, K^{*}\right)$ and $\xi_{2} \pi_{1}=\xi_{2}^{*}=\left(G_{2}^{*}, H_{2}^{*}, K_{2}^{*}\right)$. By 2.9 in [A2, $X_{i} \unlhd X$ and $\psi_{i}=l\left(\pi_{i}\right): X \rightarrow X^{i}=X / X_{i}$ is an isomorphism. As $X_{2} \unlhd X, X_{2} \psi_{1} \unlhd X^{1}$ by (1.1), and then by another application of (1.1), the preimage $Y$ of $X_{2} \psi_{1}$ in $X$ under $\psi_{1}$ is also normal in $X$. By 2.9.3 in [A2, there is a normal subfolder $\mu=\left(G_{Y}, H_{Y}, K_{Y}\right)$ of $\xi$ with $l(\mu)=Y$. As $Y \psi_{1}=X_{2} \psi_{1}$, $K_{Y}^{*}=K_{2}^{*}$, so $s_{\xi^{*}}\left(K_{2}^{*}\right)=s_{\xi^{*}}\left(K_{Y}^{*}\right)$ is a subenvelope of $\xi^{*}$ and hence as $G_{2}^{*}=H_{2}^{*} K_{2}^{*}$, $\xi_{2}^{*}$ is a subfolder of $\xi^{*}$ by 2.1 in [A2].

Let $k_{2} \in K$, let $k \in K$, and let $g \in G$. As $\xi_{2} \unlhd \xi$ the normality condition (NC) from section 2 of A2 is satisfied by $\xi_{2}$, so there is $l \in H^{g} \cap G_{2}$ and $k_{3} \in K$ with $k_{2} k=l k_{3}$. Then $k_{2}^{*} k^{*}=l^{*} k_{3}^{*}$, so $\xi_{2}^{*}$ satisfies (NC) in $\xi^{*}$, establishing (1).

Let $\alpha: \xi^{*} \rightarrow \xi^{*} / \xi_{2}^{*}$ be the natural map and let $\beta=\pi \alpha$. Then $\beta: \xi \rightarrow \xi^{*} / \xi_{2}^{*}$ is a surjective morphism with kernel $\xi_{3}$, so $\xi_{3}$ is a normal subfolder of $\xi$, establishing (2) and (3).

Let $u, g \in G_{0}$. Then $u=h_{u} k_{u}$ with $h_{u} \in H^{g}$ and $k_{u} \in K$. As $\xi_{i}$ is a subfolder of $\xi$ for $i=1,2, h_{u} \in H_{i}^{g}$ and $k_{u} \in K_{i}$, so $h_{u} \in H_{1}^{g} \cap H_{2}^{g}=H_{0}^{g}$ and $k_{u} \in K_{1} \cap K_{2}=K_{0}$, and hence $\xi_{0}$ is a subfolder of $\xi$. Similarly if $k_{0} \in K_{0}$ and $k \in K$, then $k_{0} k=l k_{4}$ for some $l \in H^{g}$ and $k_{4} \in K$, and as $\xi_{i} \unlhd \xi$ for $i=1,2, l \in H_{i}^{g}$, so $l \in H_{0}^{g}$ and hence $\xi_{0}$ satisfies (NC) in $\xi$. This establishes (4).

Of course

$$
\bar{G}_{3}=\bar{G}_{2} \times \bar{G}_{2}=\bar{H}_{1} \bar{K}_{1} \times \bar{H}_{2} \bar{K}_{2}=\left(\bar{H}_{1} \times \bar{H}_{2}\right)\left(\bar{K}_{1} \times \bar{K}_{2}\right),
$$

with $H_{1} H_{2} \leq H_{3}$. Let $a_{i} \in K_{i}, i=1,2$. Then $a_{1} a_{2}=h k, h \in H_{3}, k \in K_{3}$. Also $a_{2}^{*}=a_{1}^{*} a_{2}^{*}=h^{*} k^{*}$, so $h^{*}=1$; that is, $h \in H_{1}$. By symmetry $h \in H_{2}$, so $\bar{h}=1$ and hence $\bar{K}_{1} \times \bar{K}_{2} \subseteq \bar{K}_{3}$. Therefore $\bar{H}_{3}=\bar{H}_{1} \times \bar{H}_{3}$ and $\bar{K}_{3}=\bar{K}_{1} \times \bar{K}_{2}$, establishing (5). 
Finally applying the functor $l$ to (5), we obtain (6).

Given a set $\pi$ of primes, define a finite loop $X$ to be a $\pi$-loop if $\pi(|X|) \subseteq \pi$.

(2.2) Assume $X$ is finite and $\pi$ is a set of primes. Then:

(1) $X$ has a largest normal $\pi$-subloop $O_{\pi}(X)$.

(2) There is a normal subfolder $\xi_{\pi}=\left(G_{\pi}, H_{\pi}, K_{\pi}\right)$ of $\xi$ such that $l\left(\xi_{\pi}\right)=O_{\pi}(X)$.

Proof. Part (2) is a consequence of (1) and 2.9.3 in A2. By (2.1)(6), if $X_{1}$ and $X_{2}$ are normal $\pi$-subloops of $X$, then there is a normal $\pi$-subloop $X_{3}$ of $X$ containing $X_{1}$ and $X_{2}$, so (1) holds.

Write $O(X)$ for $O_{2^{\prime}}(X)$.

\section{RADICAL FREE BOL LOOPS}

In this section $X$ is a radical free Bol loop and $\xi=\epsilon(X)=(G, H, K)$. Adopt Notation 6.3 from [A2, and assume:

Hypothesis 3.1. $M^{+}$is a maximal subgroup of $G^{+}$containing $H\langle\tau\rangle$ and $J^{+}$is a normal subgroup of $G^{+}$contained in $M^{+}$. Set $G^{+*}=G^{+} / J^{+}, K_{M}=K \cap M^{+}$, and $\Lambda_{M}=\tau K_{M}$. Let $\Delta=G^{+} / M^{+}$and represent $G^{+}$on $\Delta$ via right multiplication.

(3.2) (1) $|K|=|\Lambda|$ and $\left|K_{M}\right|=\left|\Lambda_{M}\right|$.

(2) $\Lambda_{M}=\Lambda \cap M^{+}$.

(3) $|\Lambda|=|G: M|\left|\Lambda_{M}\right|$.

(4) Let $\lambda_{i}^{*}, 1 \leq i \leq r$, be representatives for the orbits of $G^{*}$ on $\Lambda^{*}, m_{i}=\left|\lambda_{i}^{*} G^{*}\right|$, $n_{i}=\left|\Lambda \cap \lambda_{i} J^{+}\right|$, and $n_{0}=\left|\Lambda \cap J^{+}\right|$. Then

$$
|\Lambda|=n_{0}+\sum_{i=1}^{r} n_{i} m_{i} .
$$

(5) If $\left|G^{+}: M^{+}\right|$is odd, then $n_{0}=0$ and each member of $\Lambda$ fixes a unique point of $\Delta$.

Proof. As the map $k \mapsto \tau k$ is a bijection of $K$ with $\Lambda$ and $\tau \in M^{+},(1)$ and (2) hold. The proof of (3) is straightforward and is the same as that of 12.5.1 in A2]. Similarly the proofs of (4) and (5) are essentially the same as those of parts (2) and (3) of 12.5 in A2, but we repeat the details for completeness:

Let $\Lambda_{i}=\left\{\lambda \in \Lambda: \lambda^{*} \in \lambda_{i}^{* G}\right\}$ and let $\Lambda_{0}=\Lambda \cap J^{+}$. Then $\left\{\Lambda_{i}: 0 \leq i \leq r\right\}$ is a partition of $\Lambda$ with $\left|\Lambda_{0}\right|=n_{0}$ and $\left|\Lambda_{i}\right|=n_{i} m_{i}$ for $1 \leq i \leq r$. Thus (4) holds.

Finally assume $\alpha=\left|G^{+}: M^{+}\right|$is odd. Then by Sylow's Theorem we may choose $\lambda_{i} \in M^{+}$. Set

$$
t_{i}=\left|\lambda_{i}^{* G} \cap M^{+*}\right| .
$$

Then arguing as in the proof of (4),

$$
\left|\Lambda_{M}\right|=n_{0}+\sum_{i=1}^{r} n_{i} t_{i}
$$

Therefore by (3), (4), and $(*)$,

$$
n_{0}+\sum_{i=1}^{r} n_{i} m_{i}=|\Lambda|=\alpha\left|\Lambda_{M}\right|=\alpha n_{0}+\sum_{i=1}^{r} \alpha n_{i} t_{i},
$$


$\mathrm{SO}$

$$
(\alpha-1) n_{0}+\sum_{i=1}^{r} n_{i}\left(\alpha t_{i}-m_{i}\right)=0
$$

We next claim:

For each $1 \leq i \leq r, \alpha t_{i} \geq m_{i}$, with equality iff each $\lambda \in \Lambda_{i}$ fixes a unique point of $\Delta$.

Namely each $\lambda_{i} \in \Lambda_{i}^{*}$ is in some conjugate $M^{+* g}$ of $M^{+*}$, and $\left|M^{+* g} \cap \Lambda_{i}\right|=t_{i}$, so as

$$
\left|M^{+* G}\right|=\left|G^{+}: N_{G}\left(M^{+}\right)\right|=\left|G: M^{+}\right|=\alpha,
$$

$m_{i}=\left|\Lambda_{i}^{*}\right| \leq \alpha t_{i}$ with equality iff each $\lambda^{*}$ is contained in a unique conjugate of $M^{+*}$ iff $\lambda$ fixes a unique point of $\Delta$.

Finally by (!):

$$
\begin{aligned}
& \sum_{i=1}^{r} n_{i}\left(\alpha t_{i}-m_{i}\right) \geq 0 \text { with equality iff each } \lambda \in \Lambda-\Lambda_{0} \\
& \text { fixes a unique point of } \Delta .
\end{aligned}
$$

As $\alpha>1$ we conclude from (**) and (!!) that (5) holds.

(3.3) Adopt the notation of (3.2), assume $1 \leq n_{0}$ is a power of 2 , and $p$ is an odd prime such that $m_{i} \equiv 0 \bmod p$ for all $1 \leq i \leq r$. Then $H$ contains a Sylow p-subgroup of $G$.

Proof. By $(3.2)(4),|\Lambda| \equiv n_{0} \bmod p$, so as $1 \leq n_{0}$ is a power of 2 and $p$ is odd, $|\Lambda|$ is relatively prime to $p$. Thus as $|G: H|=|\Lambda|$, the lemma follows.

\section{The Proof of Theorem 2}

In this section $X$ is a Bruck loop and $u, v \in X$.

(4.1) (1) The map $x \mapsto R(x)$ is an isomorphism of $\langle v\rangle$ with $\langle R(v)\rangle$.

(2) For each integer $n,(u \circ v) \circ v^{n}=u \circ v^{n+1}$.

Proof. Part (1) is well known; cf. 6.8 in [A1]. By (1),

$$
(u \circ v) \circ v^{n}=u R(v) R\left(v^{n}\right)=u R\left(v^{n+1}\right)=u \circ v^{n+1},
$$

establishing (2).

(4.2) $(u \circ v)^{2}=\left(v \circ u^{2}\right) \circ v$.

Proof. This appears in Lemma 1 in [G2], but we supply a proof for completeness: Let $w \in X$, and in the Bol identity (Bol), specialize $y$ to $w^{2}$ and $z$ to $w^{-1} \circ x^{-1}$ to get

$$
z \circ\left(\left(x \circ w^{2}\right) \circ x\right)=\left(\left(\left(w^{-1} \circ x^{-1}\right) \circ x\right) \circ w^{2}\right) \circ x=\left(w^{-1} \circ w^{2}\right) \circ x=w \circ x,
$$

using (4.1)(2). Next as $X$ is AIP,

$$
z \circ(w \circ x)^{2}=(w \circ x)^{-1} \circ(w \circ x)^{2}=w \circ x,
$$

so cancelling $z$ from $z \circ\left(\left(x \circ w^{2}\right) \circ x\right)=z \circ(w \circ x)^{2}$, we obtain the lemma.

(4.3) Assume $k$ is a positive integer such that $u^{2^{j}}$ commutes with $v^{2^{j-1}}$ for each $1 \leq j \leq k$. Then $(u \circ v)^{2^{i}}=u^{2^{i}} \circ v^{2^{i}}$ for each $0 \leq i \leq k$. 
Proof. The lemma is trivial if $i=0$. When $i=1,(4.1)(2)$ and (4.2) say

$$
(u \circ v)^{2}=\left(v \circ u^{2}\right) \circ v=\left(u^{2} \circ v\right) \circ v=u^{2} \circ v^{2} .
$$

Finally complete the proof by induction on $i$, using the validity of the lemma at $i=1$.

(4.4) If $v \in\langle u \circ v\rangle$, then $R(u) R(v)=R(u \circ v)=R(v \circ u)=R(v) R(u)$.

Proof. As $v \in\langle u \circ v\rangle, u=(u \circ v) \circ v^{-1} \in\langle u \circ v\rangle$ by (4.1)(2). Then the lemma follows from $(4.1)(1)$.

With these lemmas in hand, we can prove Theorem 2. Let $x, y \in X$ with $|x|=2^{n}$ and $|y|$ odd. We prove

$$
R(x) R(y)=R(x \circ y)=R(y \circ x)=R(y) R(x)
$$

by induction on $n$. Observe $(*)$ implies $x \circ y=y \circ x$, as $R$ is injective.

When $n=0,(*)$ is trivial. Assume $n>0$ and $(*)$ holds for $i<n$. Then as $\left|x^{2}\right|=2^{n-1}$, each element of $\left\langle x^{2}\right\rangle$ commutes with each element of $\langle y\rangle$ by the induction assumption. Therefore by (4.3), $(x \circ y)^{2^{n}}=x^{2^{n}} \circ y^{2^{n}}=y^{2^{n}}$, so $y^{2^{n}} \in$ $\langle x \circ y\rangle$. Then as $|y|$ is odd, $y \in\langle x \circ y\rangle$, so $(*)$ holds by (4.4).

This completes the proof of Theorem 2 .

\section{BRUCK LOOPS}

(5.1) Let $X$ be a loop with envelope $\xi=(G, H, K)$. Then the following are equivalent:

(1) $X$ is a Bruck loop.

(2) $X$ is an $A_{r}$-loop and $X$ is radical free.

(3) $H$ acts via conjugation on $K$ and $\Xi_{K}(G)=1$.

(4) $\Xi_{K}(G)=1$ and $H \leq C_{G}\left(\tau_{X}\right)$.

Proof. Parts (1) and (2) are equivalent by 6.6 in A2. Assume (2). Then $X$ is radical free, so $\Xi_{K}(G)=1$ by definition. As $X$ is an $A_{r}$-loop, $H$ acts on $K$ by 4.1 in [A2, so (3) holds. The proof of 6.7 in [A2] shows that (3) implies (4). Finally the proof of (4) in 6.6 of [A2] shows that (4) implies $X$ is an $A_{r}$-loop; thus (4) implies (2).

A loop folder $\xi=(G, H, K)$ is a Bruck loop folder if $\xi$ is a Bol loop folder, $\Xi_{K}(\langle K\rangle)=1$, and $H$ acts on $K$ via conjugation.

In the remainder of the section assume $\xi=(G, H, K)$ is a finite Bruck loop folder. We adopt the following notational conventions:

Notation 5.2. As $\Xi_{K}(\langle K\rangle)=1$, from 5.1.3.c in A2, there is a unique automorphism $\tau=\tau_{\xi}$ of $\langle K\rangle$ such that $\tau^{2}=1$ and $K \subseteq K(\tau)$. As $H$ acts on $K, H \cap\langle K\rangle$ centralizes $\tau$ by the uniqueness of $\tau$. As $\xi$ is a loop folder, $K$ is a set of coset representatives for $H$ in $G$, so as $\tau$ centralizes $H \cap\langle K\rangle$ there is a unique extension of $\tau$ to $G$ defined by $\tau: h k \mapsto h k^{\tau}$ for $h \in H$ and $k \in K$.

Form the semidirect product $G^{+}=G\langle\tau\rangle$ of $G$ by $\tau$ and let $\Lambda=\tau K \subseteq G^{+}$. By 5.1 in A2, $\Lambda$ is $\langle K\rangle$-invariant, so as $H$ centralizes $\tau$ and acts on $K$, and as $G=H K, \Lambda$ is also $G$-invariant.

Let $G_{\tau}^{+}=C_{G^{+}}(\tau), G_{\tau}=C_{G}(\tau)$, and $K_{\tau}=C_{K}(\tau)$. Set $\xi_{\tau}=\left(G_{\tau}, H, K_{\tau}\right)$.

For $U \subseteq H$, let $K_{U}=C_{K}(U), G_{U}=N_{G}(U), H_{U}=N_{H}(U)$, and $\xi_{U}=$ $\left(G_{U}, H_{U}, K_{U}\right)$. 
(5.3) (1) $\xi$ is an $A_{r}$-loop folder; that is, $H$ acts on $K$ via conjugation.

(2) For each $k \in K, H \cap H^{k}=C_{H}(k)$.

(3) $H$ controls $G$-fusion in $H$.

(4) If $\mu=\left(G_{\mu}, H_{\mu}, K_{\mu}\right)$ is a subfolder of $\xi$, then $\mu$ is a Bruck loop folder, $\tau$ acts on $G_{\mu}$, and $\tau_{\mu}=\tau_{\mid G_{\mu}}$.

(5) Suppose $\pi: \xi \rightarrow \eta=\left(G_{\eta}, H_{\eta}, K_{\eta}\right)$ is a surjective homomorphism of loop folders, and let $\xi_{0}=\left(G_{0}, H_{0}, K_{0}\right)=\operatorname{ker}(\pi)$. Then $\tau$ acts on $G_{0}, \eta$ is a Bruck loop folder, $\tau_{\eta}=\tau^{\prime}$, where $\tau^{\prime}: G_{\eta} \rightarrow G_{\eta}$ is defined by $\tau^{\prime}: g \pi \mapsto g \tau \pi$, and $\tau^{\prime}$ is the unique $\tau^{*}: G_{\eta} \rightarrow G_{\eta}$ such that $\tau \pi=\pi \tau^{*}$.

Proof. Part (1) follows from the definition of Bruck folders. Then (1) and 4.3 in A2 imply (2) and (3).

Assume the hypotheses of (4). Then $K_{\mu} \subseteq K \subseteq K(\tau)$, so $\tau$ acts on $K_{\mu}$, and $H_{\tau} \leq H \leq C_{G}(\tau)$, so $\tau$ acts on $H_{\mu}$. Therefore $\tau$ acts on $G_{\mu}=H_{\mu} K_{\mu}$. By 6.2 in [A2], $\mu$ is a Bol loop folder, and by construction $H_{\mu} \subseteq C_{G_{\mu}}(\tau)$ and $K_{\mu} \subseteq K(\tau)$, so by 5.2 in $\left[\mathrm{A} 2, \tau_{\mid\left\langle K_{\mu}\right\rangle}=\tau_{\left\langle K_{\mu}\right\rangle}\right.$, completing the proof of (4).

Finally assume the hypotheses of (5). Then $\xi$ is a normal subfolder of $\xi$, so $\tau$ acts on $G_{0}$ by (4) and hence induces $\tau^{\prime}: G_{\eta} \rightarrow G_{\eta}$ defined by $\tau^{\prime}: g \pi \mapsto g \tau \pi$; further $\tau^{\prime}$ is the unique map $\tau^{*}: G_{\eta} \rightarrow G_{\eta}$ such that $\tau \pi=\pi \tau^{*}$. As $\pi: K \rightarrow K_{\eta}$ is surjective and $K \subseteq K(\tau)$, this implies $K_{\eta} \subseteq K\left(\tau^{\prime}\right)$, and similarly $\tau^{\prime}$ centralizes $H_{\eta}$, so by 5.2 in [A2], $\Xi_{K_{\eta}}\left(\left\langle K_{\eta}\right\rangle\right)=1$ and $\tau^{\prime}=\tau_{\eta}$. Thus (5) holds.

(5.4) (1) $\xi_{\tau}$ is a Bruck loop folder.

(2) $X_{\tau}=l\left(\xi_{\tau}\right)$ is of exponent 2 .

(3) $K_{\tau}$ is $G_{\tau}$-invariant and $\Lambda_{\tau}=C_{\Lambda}(\tau)=\tau K_{\tau}$.

Proof. By 6.6.5 in A2, $\xi_{\tau}$ is a subfolder of $\xi$ and (2) holds. Then (1) follows from (5.3)(4). For $g \in G_{\tau}$ and $k \in K_{\tau}, k=\tau \lambda$ for some $\lambda \in \Lambda_{\tau}$, so $k^{g}=\tau \lambda^{g} \in \tau \Lambda_{\tau}=K_{\tau}$ as $\Lambda$ is $G$-invariant, so $K_{\tau}$ is $G_{\tau}$-invariant. Thus (3) holds.

(5.5) Let $U \subseteq H$ and $X=l(\xi)$. Then:

(1) $\xi_{U}$ is a Bruck loop folder.

(2) $X_{U}=F x_{X}(U)$ is a Bruck subloop of $X$ with $l\left(\xi_{U}\right)=X_{U}$.

(3) $K_{U}=N_{K}(U)$.

(4) $\left\langle K_{U}\right\rangle$ is transitive on $X_{U}$.

(5) $\Lambda_{U}=N_{\Lambda}(U)=C_{\Lambda}(U)=\tau K_{U}$.

(6) If $h \in H$ and $h^{2} \neq 1$, then $h$ is not inverted by any member of $\Lambda$. In particular $\tau$ inverts no conjugate of $h$.

Proof. By (5.3)(1), $\xi$ is an $A_{r}$-loop folder, so by (4.3)(3) in [A2], $\xi_{U}$ is a subfolder of $\xi$. Thus (1) follows from (5.3)(4). By parts (1) and (2) of 4.3 in [A2, $X_{U}$ is a subloop of $X$ with $l\left(\xi_{U}\right)=X_{U}$. Then as $\xi_{U}$ is a Bruck folder, $X_{U}$ is a Bruck loop, so (2) holds. Parts (3) and (4) follow from parts (4) and (6) of 4.3 in [A1.

Next $N_{\Lambda}(U)=\tau N_{K}(U)=\tau K_{U}$, so (5) follows from (3). Then (6) follows from (5).

(5.6) Assume $\xi$ is an envelope, $Q \leq G$ with $Q \unlhd G^{+}$, and set $G^{+*}=G^{+} / Q$. Then the following are equivalent:

(1) $\left|k^{*}\right|$ is odd for each $k \in K$.

(2) $\left|\tau^{*} \lambda^{*}\right|$ is odd for each $\lambda \in \Lambda$.

(3) $\left|G^{*}\right|$ is odd. 
Proof. As $K=\tau \Lambda,(1)$ and (2) are equivalent. Trivially (3) implies (2). Finally if (2) holds, then $\Lambda^{*}=\tau^{* G}$ and by Glauberman's $Z^{*}$-Theorem [G1], $G^{*}=\left\langle K^{*}\right\rangle=$ $\left\langle\tau^{*} \Lambda^{*}\right\rangle$ is of odd order.

Recall the definition of a $\pi$-loop from section 2. In particular a loop $X$ is a 2-loop if $|X|$ is a power of 2 , and $X$ is a $2^{\prime}$-loop if $|X|$ is odd.

(5.7) Assume $\xi$ is an envelope and set $X=l(\xi)$. Then:

(1) The following are equivalent:

(a) $X$ is a 2-loop.

(b) $G$ is a 2-group.

(c) $\alpha \beta$ is a 2-element for all $\alpha, \beta \in \Lambda$.

(2) The following are equivalent:

(a) $X$ is a $2^{\prime}$-loop.

(b) $|G|$ is odd.

(c) $|k|$ is odd for all $k \in K$.

Proof. As $|X|=|K|=|G: H|$, (b) implies (a) and (c) in (1) and (2).

Assume $X$ is a 2-loop. Then $|G: H|$ is a power of 2 , so for each odd prime $p$ and each element $g$ of order $p$ in $G, g$ is conjugate to an element of $H$. Thus no member of $\Lambda$ inverts $g$ by (5.5)(6). Hence by the Baer-Suzuki Theorem (cf. 39.6 in [FGT]), $\Lambda \subseteq O_{2}\left(G^{+}\right)$, so $K=\tau \Lambda \subseteq O_{2}(G)$. Therefore $G=\langle K\rangle$ is a 2-group. Similarly if (1c) holds, then no member of $\Lambda$ inverts a nontrivial element of odd order, so the same argument shows $G$ is a 2-group, completing the proof of (1).

Assume $X$ is a $2^{\prime}$-loop. As each $k \in K^{\#}$ is fixed point free on $X$, while $\langle k\rangle \subseteq K$ by 5.1 in [A2, $k$ is semiregular on $X$, so $|k|$ divides $|X|$ and hence $|k|$ is odd. Therefore (2a) implies (2c), while (2c) implies (2b) by 5.6, completing the proof of (2).

(5.8) Let $L$ be a group of odd order and let $t$ be an involutory automorphism of $L$. Then $\mu=\left(L, C_{L}(t), K_{L}(t)\right)$ is a Bruck loop folder, where $K_{L}(t)=\left\{l \in L: l^{t}=\right.$ $\left.l^{-1}\right\}$.

Proof. Let $C=C_{L}(t)$ and $K=K_{L}(t)$. The map $\sigma: C g \mapsto[t, g]$ is a welldefined injection of $L / C$ into $K$. Further as $|L|$ is odd, for $k \in K, t k \in t^{L}$, so $k \in t t^{L} \subseteq \sigma(L / C)$. Thus $\sigma$ is a bijection, so $|L: C|=|K|$. Finally if $a, b, c \in t^{G}$ with $1 \neq x=a b \in C_{G}(c)$, then $\langle a\rangle$ and $\langle c\rangle$ are Sylow in the normalizer of $X=\langle x\rangle$, so there is $g \in N_{G}(X)$ with $a^{g}=c$ by Sylow's Theorem. This is impossible as $a$ inverts $X$, while $c$ centralizes $X$. Thus $\mu$ is a Bol loop folder and $\Xi_{K}(\langle K\rangle)=1$ by the equivalence of parts (1) and (6) of 6.4 in $\mathrm{A2}$. Then by construction, $\mu$ is a Bruck folder.

(5.9) Assume $G_{0} \unlhd G$, set $G^{*}=G / G_{0}$, and assume $\left|k^{*}\right|$ is odd for each $k \in K$. Let $H_{0}=H \cap G_{0}, K_{0}=K \cap G_{0}$, let $\xi_{0}=\left(G_{0}, H_{0}, K_{0}\right)$, and let $\pi: G \rightarrow G^{*}$ be the natural map. Then:

(1) $G^{*}$ is of odd order.

(2) $\xi_{0}$ is a normal subfolder of $\xi$ and $\pi: \xi \rightarrow \xi / \xi_{0}=\left(G^{*}, H^{*}, K^{*}\right)$ is a surjective morphism of loop folders with $\xi_{0}=\operatorname{ker}(\pi)$.

(3) $l(\xi) / l\left(\xi_{0}\right) \cong l\left(\xi / \xi_{0}\right)$ is a $2^{\prime}$-loop.

Proof. Part (3) follows from (1), (2), 2.7 in A2], and (5.7)(2); part (1) follows from (5.6). 
Let $t$ be the involutory automorphism of $G^{*}$ induced by $\tau$ as in (5.3)(5), and $K_{t}=K_{G^{*}}(t)$. By (5.8), $\xi^{*}=\left(G^{*}, C_{G^{*}}(t), K_{t}\right)$ is a Bruck loop folder. Further $H^{*} \leq C_{G^{*}}(t), K^{*} \subseteq K_{t}$, and $G^{*}=H^{*} K^{*}$, so it follows that $H^{*}=C_{G^{*}}(t)$ and $K^{*}=K_{t}$. Thus $\xi^{*}=\left(G^{*}, H^{*}, K^{*}\right)$ is a loop folder and $\pi: \xi \rightarrow \xi^{*}$ is a surjective morphism of folders, so $\xi_{0}=\operatorname{ker}(\pi)$ is a normal subloop of $\xi$ and $\xi^{*}=l(\xi) / l\left(\xi_{0}\right)$ by definition of the notation in 2.6 of A2. That is (2) holds.

(5.10) Assume $|G|$ is odd and let $X=l(\xi)$. Then:

(1) $\Lambda=\tau^{G}, H=C_{G}(\tau)$, and $K=K(\tau)$.

(2) The map $\varphi: J \mapsto\left(J, C_{J}(\tau), K_{J}(\tau)\right)$ is a bijection between the set $\mathcal{J}$ of $\tau$-invariant subgroups $J$ of $G$ and the set $\mathcal{F}$ of subfolders of $\xi$.

(3) Under the bijection $\varphi$, normal subgroups of $G$ correspond to normal subfolders of $\xi$.

(4) The map $Y \mapsto\langle\kappa(Y)\rangle$ is a bijection between the set of subloops of $X$ and the set $\mathcal{L}$ of $L \in \mathcal{J}$ such that $L=[L, \tau]$.

(5) $G$ and $X$ are solvable.

Proof. The proof of (5.9) in the special case where $G_{0}=1$ shows that (1) holds.

By (5.8), $\varphi$ is a map from $\mathcal{J}$ into $\mathcal{F}$, and by construction, $\varphi$ is injective. If $\mu=\left(J, H_{J}, K_{J}\right) \in \mathcal{F}$, then $J$ is $\tau$-invariant and $\mu$ is a Bruck folder with $\tau_{\mu}=\tau_{\mid J}$ by (5.3)(4). Thus $H_{J}=C_{J}(\tau)$ and $K_{J}=K_{J}(\tau)$ by (1). Hence $\varphi$ is a surjection, completing the proof of (2). If $J \unlhd G$, then $\varphi(J) \unlhd \xi$ by (5.9), so (3) also holds.

Let $\mathcal{Y}$ be the set of subloops of $X$ and let $\psi$ be the map in (4) (cf. Convention 1.9 in [A2 for the definition of $\kappa$ ). Then $\psi$ is an injection from $\mathcal{Y}$ into $\mathcal{L}$. Further if $L \in \mathcal{L}$, then $\varphi(L) \in \mathcal{F}$, so $\phi(L)=l(\varphi(L)) \in \mathcal{Y}$, and hence $\phi$ is a map from $\mathcal{L}$ to $\mathcal{Y}$. Next $\kappa(Y)=K \cap \psi(Y)$, so $\kappa(Y)$ is the set of translations of $\varphi(\psi(Y))$, and hence $Y=l(\varphi(\psi(Y)))=\phi(\psi(Y))$. Similarly $K_{L}\left(\tau_{\mid L}\right)=\kappa(\phi(L))$, so $L=\psi(\phi(L))$, completing the proof of (4).

By the Odd Order Theorem [FT], $G$ is solvable. Thus a minimal normal subgroup $L$ of $G$ is an elementary abelian $p$-group for some prime $p$. By (3), $\varphi(L) \triangleleft \xi$, so by 2.9 in [A1], $\phi(L) \unlhd X$. As $L$ is abelian, $H \cap L \unlhd L$, so $\phi(L) \cong L / H \cap L$ by 2.10 in A1]. By induction on the order of $X, X / \phi(X)$ is solvable, so $X$ is solvable.

(5.11) $D(G)=\langle K\rangle \unlhd G$.

Proof. This holds as $G=H K$ and $H$ acts on $K$ via conjugation.

(5.12) If $X=O_{2}(X) \times O(X)$, then $X$ is solvable.

Proof. By (5.10)(5), $O(X)$ is solvable, while by 7.4 in $\mathrm{A} 2$ and $(5.7)(1), O_{2}(X)$ is solvable.

(5.13) The following are equivalent:

(1) $X$ is a 2-element loop.

(2) $k$ is a 2-element for each $k \in K$.

(3) $\tau \in O_{2}\left(G^{+}\right)$.

Proof. Parts (1) and (2) are equivalent by (4.1)(1). If (2) holds, then $\tau \tau^{g}$ is a 2-element for each $g \in G$, so (3) holds by the Baer-Suzuki Theorem (cf. 39.6 in [FGT]). Conversely if (3) holds, then for each $\lambda \in \Lambda, \tau \lambda \in\langle\lambda\rangle O_{2}\left(G^{+}\right)$, so $k=\tau \lambda$ is a 2-element; that is, (3) implies (2). 


\section{The Proof of Theorem 1}

In this section we establish Theorem 1. Thus we assume $X$ is a finite Bruck loop and we set $\xi=\epsilon(X)=(G, H, K)$.

Let $X_{r}$ be the set of $r$-elements of $X$ for $r \in\left\{2,2^{\prime}\right\}, K_{r}=R\left(X_{r}\right)$, and $G_{r}=\left\langle K_{r}\right\rangle$.

(6.1) (1) For each $x \in X, x=x_{2} \circ x_{2^{\prime}}$ with $x_{r} \in X_{r} \cap\langle x\rangle$, and this expression is unique.

(2) $R(x)=R\left(x_{2}\right) R\left(x_{2^{\prime}}\right)$ with $R\left(x_{r}\right) \in K_{r}$.

(3) $G=G_{2} * G_{2^{\prime}}$ is the central product of $G_{2}$ and $G_{2^{\prime}}$; that is, $G=G_{2} G_{2^{\prime}}$ and $\left[G_{2}, G_{2^{\prime}}\right]=1$.

(4) $O^{2^{\prime}}(X) \unlhd X$.

(5) $G_{2^{\prime}} \leq O(G)$.

(6) $X_{2^{\prime}}=O^{2}(X)=O(X) \unlhd X$ and $X / O(X)$ is a 2-element loop.

Proof. Parts (1) and (2) follow from (4.1)(1) and the corresponding statement for groups. Then (3) follows from (1), (2), and Theorem 2.

By (3), $G_{2} \unlhd G$. Let $G^{*}=G / G_{2}, J_{r}=K \cap G_{2}$, and $Y=R^{-1}\left(J_{2}\right)$. For $R(x) \in J_{2}, R(x)=R\left(y_{1}\right) \cdots R\left(y_{n}\right)$ with $y_{i} \in X_{2}$. Define $x_{1}=y_{1}$ and for $i>1$ define $x_{i}=x_{i-1} \circ x_{i}$ recursively. Then $x_{n} \in X_{2}$ and $x=1 R(x)=1 R\left(y_{1}\right) \cdots R\left(y_{n}\right)=$ $1 R\left(x_{n}\right)=x_{n}$, so $Y=O^{2^{\prime}}(X)$.

Each element of $K^{*}$ is of odd order, so (5.9) tells us that $G^{*}$ is of odd order and $Y=O^{2^{\prime}}(X)$ is a normal subloop of $X$. Let $L=G_{2^{\prime}}$ and $U=L \cap G_{2}$. Then $L^{*} \cong L / U$ is of odd order, and hence solvable by the Odd Order Theorem [FT]. Also $U \leq Z(L)$ by (3), so $L$ is solvable and hence $L=L_{0} U$, where $L_{0}$ is a Hall $2^{\prime}$-subgroup of $L$ by Phillip Hall's Theorem 18.5 in [FGT]. Then as $U \leq Z(L)$, $L=O^{2}(L)=L_{0}$ is of odd order, establishing (5).

By (5.8), $\xi_{2^{\prime}}=\left(L, C_{L}(\tau), K_{2^{\prime}}\right)$ is a subfolder of $\xi$, so by 1.9 in [A2, $X_{2^{\prime}}=$ $R^{-1}\left(K_{2^{\prime}}\right)$ is a subloop of $X$. Then by definition, $X_{2^{\prime}}=O^{2}(X)$. Let $u \in K_{2^{\prime}}$ and $v \in K$. By (2), $v=b a$ with $b \in K_{2^{\prime}}$ and $a \in K_{2}$, so $u v=u b a$. As $\xi_{2^{\prime}}$ is a subfolder, $u b=h b^{\prime}$ with $h \in C_{L}(\tau)$ and $b^{\prime} \in K_{2^{\prime}}$. By Theorem $2, b^{\prime} a=k^{\prime} \in K$, so $u v=h v^{\prime}$; that is, the normality condition (NC) of section 2 of $\mathrm{A} 2$ is satisfied. Hence (6) follows from 2.9 in $\mathrm{A} 2$.

We are now in a position to prove Theorem 1. We apply (2.1) to $G_{2^{\prime}}$ and $G_{2}$ in the roles of the groups " $G_{1}$ " and " $G_{2}$ " in that lemma. From the proof of (6.1), the subfolders $\xi_{r}=\left(G_{r}, H \cap G_{r}, J_{r}\right)$ are normal with $J_{2^{\prime}}=O(X)$ and $J_{2}=O^{2^{\prime}}(X)$. By (6.1)(3), $G=G_{1} G_{2}=G_{3}$. Thus by (2.1), $\bar{G}=\bar{G}_{1} \times \bar{G}_{2}$ and $X / X_{0} \cong X_{1} / X_{0} \times X_{2} / X_{0}=O^{2^{\prime}}(X) / X_{0} \times O(X) / X_{0}$. Also $X_{0}=R^{-1}(J)$, where $J=K_{2^{\prime}} \cap G_{2}$. By (6.1)(3), $J \leq Z(G)$. For $k \in K, k=b a$ with $a \in K_{2}$, $b \in K_{2^{\prime}}$. As $\tau$ inverts $j$ and $b$ and $j \in Z(G), \tau$ inverts $j b$, so $j b \in K_{2^{\prime}}$ by (5.8). Thus $j k=j b a \in K$ by Theorem 2. Hence $j K \subseteq K$, so $R^{-1}(j) \in Z(X)$; that is, $X_{0} \leq Z(X)$.

We have established the first four statements in Theorem 1 , so it remains to establish the fifth. Thus we may assume $X$ is solvable. Moreover we assume $X$ is a counterexample of minimal order to part (5) of Theorem 1 . Therefore $O^{2^{\prime}}(X) \neq O_{2}(X)$, so $O^{2^{\prime}}(X)$ is not a 2-loop. Also each proper section $Y$ of $X$ is solvable, so by minimality of $X, Y=O_{2}(Y) \times O(Y)$ and $G_{Y}=O_{2}\left(G_{Y}\right) \times O\left(G_{Y}\right)$, where $G_{Y}$ is the enveloping group of $Y$. In particular $X=O^{2^{\prime}}(X)$ is not a 2-loop and $G=O^{2^{\prime}}(G)$ is not a 2-group. 
Suppose $O(X) \neq 1$. By minimality of $X, Y=X / O(X)$ is a 2-loop and $G_{Y}$ is a 2-group. Let $U=R(O(X))$ and $G^{*}=G / U$. As $O(X) \leq Z(X), \xi_{0}=(U, 1, U)$ is a normal subfolder of $\xi, \xi^{*}=\left(G^{*}, H^{*}, K^{*}\right)=\xi / \xi_{0}$, and $Y \cong l\left(\xi / \xi_{0}\right)$, so $G_{Y}=$ $G^{*} / \operatorname{ker}_{H^{*}}\left(G^{*}\right)$. By $(3.3)(2), \operatorname{ker}_{H^{*}}\left(G^{*}\right) \leq Z\left(G^{*}\right)$, so as $G_{Y}$ is a 2-group and $U \leq$ $Z(G), G$ is solvable and $G=T Z(G)$ for $T \in S y l_{2}(G)$ by coprime action (cf. 18.7.4 in [FGT]). Thus $G=O^{2^{\prime}}(G)=T$, contradicting that $G$ is not a 2-group.

Let $Y$ be a maximal normal subloop of $X$. As $O(X)=1$ and $Y=O_{2}(Y) \times O(Y)$ with $O(Y)=O^{2}(Y) \leq O^{2}(X)=O(X), Y$ is a 2-loop. As $X$ is a solvable 2-element loop and $Y$ a maximal normal subloop of $X, X / Y \cong \mathbf{Z}_{2}$. Thus $|X|=2|Y|$ is a power of 2, so $X$ is a 2-loop, for our final contradiction.

\section{The Proof of Theorem 3}

In this section we establish Theorem 3 .

Assume $X$ is an M-loop and let $\xi=\epsilon(X)=(G, H, K)$.

(7.1) (1) $X$ is simple.

(2) $O(X)=O_{2}(X)=1$.

(3) $X$ is a 2-element loop.

(4) Theorem 3 holds if $X$ is of exponent 2.

Proof. If $Y$ is a proper nontrivial normal subloop of $X$, then $Y$ and $X / Y$ are proper sections of $X$, and hence are solvable; but then $X$ is also solvable, contradicting the hypothesis that $X$ is an $M$-loop. Therefore (1) holds. Then (2) follows from (1) and (5.12). Finally if $X$ is of exponent 2, then $X$ is an N-loop, as defined in [A2], so Theorem 3 holds in this case by the Main Theorem of A2.

By $(7.1)(3), X$ is a 2-element loop. We can repeat many of the lemmas from section 12 of [A2], proved there under the stronger hypothesis that $X$ is of exponent 2. By (7.1)(4), we may assume $X$ is not of exponent 2. Adopt Notation 5.2, and for $U \leq G$ set $D(U)=\langle K \cap U\rangle$. For $U \leq H$, let $D_{U}=D\left(G_{U}\right)$.

(7.2) Assume $p$ is an odd prime divisor of $|H|$ and let $1 \neq P$ be a p-subgroup of H. Then:

(1) $H$ contains a Sylow p-subgroup of $H$.

(2) No member of $\Lambda$ inverts an element of order $p$.

(3) $\left|N_{G}(P): N_{H}(P)\right|$ is a power of 2 .

Proof. As $\xi_{P}$ is a proper subfolder, $G_{P}=H_{P} D_{P}$, and $D_{P}$ is a 2-group as $X$ is a 2-element M-loop. Thus (3) holds. Then (3) implies (1), while (1) and (5.5)(6) imply (2).

(7.3) $|G: H|$ is not a power of 2 .

Proof. By $(7.1)(2), G$ is not a 2-group, so as $|X|=|G: H|$, the lemma follows from (5.7)(1).

During the remainder of this section we work in the following setup.

Hypothesis 7.4. $M^{+}$is a maximal overgroup of $\langle\tau\rangle H$ in $G^{+}$. Set $M=M^{+} \cap G$, $J^{+}=\operatorname{ker}_{M^{+}}\left(G^{+}\right), K_{M}=K \cap M^{+}, \Lambda_{M}=\tau K_{M}, D=D(M)$, and $G^{+*}=G^{+} / J^{+}$.

(7.5) (1) Hypothesis 3.1 is satisfied.

(2) $\mathrm{O}_{2}(G)\langle\tau\rangle=\mathrm{O}_{2}\left(G^{+}\right) \leq J$.

(3) $\left|G^{+}: M\right|$ is even. 
Proof. Visibly Hypothesis 7.4 implies Hypothesis 3.1, so (1) holds. As $X$ is a 2element loop, $\tau \in O_{2}\left(G^{+}\right)$by (5.13), so (2) holds. Finally $\tau \in \Lambda \cap J$ by (2), so (1) and (3.2)(5) imply (3).

(7.6) (1) $M=H D$ and $D$ is a 2-group.

(2) $\left|\Lambda_{M}\right|=|M: H|$ is a power of 2 .

(3) $|G: M|$ is even but not a power of 2 .

(4) $M$ and $H$ are not 2-groups.

(5) $D \unlhd G, H^{*}=M^{*}$, and $K^{*} \cap M^{*}=1$.

(6) Let $N$ be the preimage in $G$ of $F^{*}\left(G^{*}\right)$. Then $G=H N$.

(7) $J$ is a 2-group.

Proof. The proofs of (1)-(4) are the same as that of the correponding parts of 12.6 in A2. Similarly if $D \unlhd G$, then the proof of 12.6 .5 in [A2] shows that (5) holds, so suppose $D$ is not normal in $G$. Recall $D \unlhd M^{+}$by $(5.11)$, so $M^{+}=N_{G^{+}}(D)$ by maximality of $M^{+}$. Next let $D^{+}=D\langle\tau\rangle$; thus $D=D^{+} \cap G$ and $D^{+}=\left\langle\Lambda_{M}\right\rangle$, with $\Lambda_{M}=\Lambda \cap M$. Thus $D^{+}=\left\langle N_{\Lambda}\left(D^{+}\right)\right\rangle$, so taking $D^{+} \leq T^{+} \in \operatorname{Syl}_{2}\left(G^{+}\right)$, $D^{+}=\left\langle\Lambda \cap N_{T^{+}}\left(D^{+}\right)\right\rangle$and hence $D^{+}=\left\langle T^{+} \cap \Lambda\right\rangle$, so $T^{+} \leq N_{G}\left(D^{+}\right) \leq M^{+}$, contrary to $(3)$.

Now parts (6) and (7) follow as in the proof of the corresponding parts of 12.6 in $\mathrm{A} 2$.

(7.7) Suppose $1 \neq U^{*} \leq H^{*}$ is a p-group for some odd prime $p$.

(1) $H$ contains a Sylow p-group of the preimage of $U^{*}$ in $G^{*}$.

(2) $N_{G^{*}}\left(U^{*}\right)=N_{G}(P)^{*}-N_{H}(P)^{*} D\left(C_{G}\left(P^{*}\right)\right)$.

(3) The triple $G^{*}, M^{*}, K^{*}$ satisfies Hypothesis $N$ of section 10 of [A2].

(4) $K^{*}=\Lambda^{*}$.

Proof. The proofs of (1)-(3) are the same as those of the corresponding parts of 12.7 in A2. Use (3.3) in proving (3). Note as $\tau \in J, K^{*}=\Lambda^{*}$ is a union of conjugacy classes of involutions of $G^{*}$.

(7.8) $F^{*}\left(G^{*}\right)$ is a nonabelian simple group and $G^{*}=F^{*}\left(G^{*}\right) H^{*}$.

Proof. The proof is the same as that of 12.8 in $\mathrm{A} 2$.

Theorem 7.9. (1) $G^{*} \cong P G L_{2}(q)$ with $q=2^{n}+1, H^{*}$ is a Borel subgroup of $G^{*}$, and $K^{* \#}$ consists of the involutions in $G^{*}-F^{*}\left(G^{*}\right)$.

(2) $\left|K^{* \#}\right|=m=q(q-1) / 2$ and $|G: M|=q+1$.

(3) Let $n_{0}=|K \cap J|$ and $n_{1}=|K \cap a J|$ for $a \in K-J$. Then $n_{0}=|M: H|=\mid D$ : $D \cap H \mid$ is a power of $2, n_{0}=n_{1} 2^{n-1}$, and $|X|=|K|=(q+1) n_{0}=n_{1} 2^{n}\left(2^{n-1}+1\right)$.

(4) $F^{*}(G)=J=O_{2}(G)$.

Proof. The proofs of (1)-(3) are the same as the corresponding parts of 12.9 in [A2]. Note that since $\tau \in J^{+}$, for $\lambda \in \Lambda-J^{+}, \lambda=\tau a$ for some $a \in K-J^{+}$and the map $k \mapsto \tau k$ is a bijection of $K \cap a J^{+}$with $\lambda J^{+} \cap \Lambda$.

By (5.4), $G_{\tau}=H D_{\tau}$, where $D_{\tau}=D\left(G_{\tau}\right)$ and $X_{\tau}$ is of exponent 2. As we are assuming that $X$ is not of exponent $2, X_{\tau} \neq X$, so $X_{\tau}$ is a 2-loop, and hence $D_{\tau}$ is a 2-group. Thus each subgroup of $G_{\tau}$ of odd order is fused into $H$ under $D_{\tau}$. However if (4) fails, then as $\tau \in J^{+}=O_{2}\left(G^{+}\right), G^{+}=L J^{+}$, where $L=E(G)$, and $\tau$ centralizes $L$. But then $L$ centralizes $D_{\tau}$, so each subgroup of $L$ of odd order is contained in $H$. Therefore $E=O^{2}(E) \leq H$, so $E \leq \operatorname{ker}_{H}(G)=1$, a contradiction.

Observe that 7.1 and Theorem 7.9 establish Theorem 3. 


\section{The proof of Corollary 4}

In this section we prove Corollary 4. Thus we assume $X$ is a Bol loop which is also an $A_{r}$-loop. Let $\xi=\epsilon(X)=(G, H, K), G^{*}=G / \Xi_{K}(G)$, and $\hat{G}=G^{*} / \operatorname{ker}_{H^{*}}\left(G^{*}\right)$.

(8.1) (1) $\xi_{\Xi}=\left(\Xi_{K}(G), 1, \Xi_{K}(G)\right)$ is a normal subfolder of $G$.

(2) $\Xi(X)$ is a normal subloop of $X$.

(3) $\Xi(X)$ is isomorphic to the group $\Xi_{K}(G)$.

(4) $X / \Xi(X) \cong l\left(G^{*}, H^{*}, K^{*}\right)$.

(5) There is a unique automorphism $\tau$ of $G^{*}$ with $\tau^{2}=1$ and $K^{*} \subseteq K(\tau)$.

(6) $H^{*} \leq C_{G^{*}}(\tau)$.

(7) $\epsilon(X / \Xi(X)) \cong(\hat{G}, \hat{H}, \hat{K})$.

(8) $X / \Xi(X)$ is a Bruck loop.

(9) $\operatorname{ker}_{H^{*}}\left(G^{*}\right) \leq Z\left(G^{*}\right)$.

Proof. Parts (1)-(4) are the corresponding parts of 6.5 in [A2]. Then (7) is a consequence of (4) and 2.9.2 in A2]. Part (5) follows from 5.1.3 in A2]. As $X$ is an $A_{r}$-loop, $H$ acts on $K$ via conjugation by 4.1 in $\mathrm{A} 2$, so $H^{*}$ acts on $K^{*}$. Therefore (6) follows from the uniqueness of $\tau$ in (5). Thus $\tau$ acts on $\operatorname{ker}_{H^{*}}\left(G^{*}\right)$ and hence induces an automorphism $\hat{\tau}$ of $\hat{G}$ centralizing $\hat{H}$ with $\hat{K} \subseteq K(\hat{\tau})$. Therefore $\Xi_{\hat{K}}(\hat{G})=1$ by 5.1.3 in A2]. Then (8) follows from this fact and the fact that $\hat{\tau}$ centralizes $\hat{H}$, given the equivalence of parts (1) and (4) of (5.1). Finally (9) follows from 4.3 .4 in $\mathrm{A} 2$.

Assume $X$ is solvable. Then $\Xi(X)$ is solvable, so the subgroup $\Xi_{K}(G)$ is also solvable by $(8.1)(3)$. Next by $(8.1)(8), X / \Xi(X)$ is a Bruck loop, and solvable as $X$ is solvable. Then by Theorem 1 and the Odd Order Theorem [FT], the enveloping group $\bar{G}$ of $X / \Xi(X)$ is solvable, and by $(8.1)(7), \bar{G} \cong \hat{G}$. Then appealing to (8.1)(9), $G$ is solvable.

Thus to complete the proof of Corllary 3 it remains to show that $X$ is solvable if $G$ is solvable. Assume otherwise and choose a counterexample $X$ of minimal order.

As $G$ is solvable, so are $\Xi_{K}(G)$ and $\hat{G}$. Hence $\Xi(X)$ is solvable by $(8.1)(3)$. Further by $(8.1)(8), X / \Xi(X)$ is a Bruck loop, and by $(8.1)(7), \hat{G}$ is its enveloping group. Thus if $\Xi(X) \neq 1$, then $X / \Xi(X)$ is solvable by minimality of $X$, so $X$ is solvable, contrary to the choice of $X$. Therefore $X$ is radical free and a Bruck loop.

Next if $Y$ is a proper section of $X$, then by 2.9 in A1, the enveloping group of $Y$ is a section of $G$, and hence is solvable. Therefore by minimality of $X, Y$ is solvable. Thus $X$ is an M-loop. But now Theorem 3 supplies a contradiction, since $G$ is solvable.

\section{REFERENCES}

[A1] M. Aschbacher, Near subgroups of finite groups, J. Group Theory 1 (1998), 113-129. MR.1614316 (99e:20031)

[A2] M. Aschbacher, On Bol loops of of exponent 2 (to appear in J. Algebra).

[Ba] R. Baer, Nets and groups, Trans. AMS 47 (1939), 110-141. MR0000035(1:6e)

[Br] R. Bruck, A Survey of Binary Systems, Springer-Verlag, Berlin, 1971. MR0093552 (20:76)

[FT] W. Feit and J. Thompson, Solvability of groups of odd order, Pacific J. Math. 13 (1963), 755-1029. MR0166261 (29:3538)

[FGT] M. Aschbacher, Finite Group Theory, Cambridge Univ. Press, Cambridge, 1986. MR 0895134 (89b:20001)

[G1] G. Glauberman, Central elements in core free groups, J. Algebra 4 (1966), 403-420. MR0202822 (34:2681) 
[G2] G. Glauberman, On loops of odd order, I, J. Algebra 1 (1964), 374-396. MR0175991 (31:267)

[G3] G. Glauberman, On loops of odd order, II, J. Algebra 8 (1968), 393-414. MR0222198 $(36: 5250)$

Department of Mathematics, California institute of Technology, Pasadena, CaliFORNIA 91125

Department of Mathematical Sciences, Indiana University South Bend, South Bend, INDIANA 46634

Department of Mathematics and Computer Science, Wabash College, CrawfordsVILLE, INDiana 47933 